\title{
INFLUÊNCIA DE ELEMENTOS DO CLIMA NO DESPERDÍCIO DE ENERGIA EM UM SISTEMA DE IRRIGAÇÃO POR ASPERSÃO DE ALTA PRESSÃ̃ ${ }^{1}$
}

\author{
Hamilton Jorge Azevedo 2 , Salassier Bernardo ${ }^{3}$, Marcio Mota Ramos ${ }^{4}$, \\ Gilberto Chohaku Sediyama ${ }^{4} \&$ Paulo Roberto Cecon ${ }^{5}$
}

\begin{abstract}
RESUMO
Realizou-se um estudo para avaliação do desperdício de energia correspondente à porcentagem de perda de água do jato de um aspersor de alta pressão, na região Norte Fluminense, RJ. Por meio da metodologia descrita pela ASAE (1990) foi analisado o desperdício de energia em função dos seguintes elementos do clima: velocidade do vento, umidade relativa do ar e radiação solar e da pressão de serviço do aspersor. Observou-se que a umidade relativa teve maior influência no desperdício de energia (desperdício em Mega Joule por hectare hora) seguida pela velocidade do vento, pressão de serviço do aspersor e radiação solar.
\end{abstract}

Palavras-chaves: aspersão, clima, energia, perdas

\section{INFLUENCE OF WEATHER ELEMENTS ON ENERGY LOSSES IN HIGH PRESSURE SPRINKLE IRRIGATION SYSTEM}

\begin{abstract}
A study was performed to determine the energy loss corresponding to percentage of water losses in high pressure sprinkler in the Norte Fluminense region of Rio de Janeiro. A field experiment was carried out according to ASAE (1990) methodology. Energy loss was analyzed as a function of the following independent variables: wind speed, air relative humidity, solar radiation and pressure head of sprinkler. Results obtained showed that relative humidity of air had the most influence in the energy loss (energy loss in Mega Joule per hectare hour) followed by wind speed, sprinkler pressure head and solar radiation.
\end{abstract}

Key words: sprinkle, climate, energy, losses

\section{INTRODUÇÃO}

O alto custo da energia tem reduzido a margem de lucro da agricultura irrigada com sistemas de irrigação por aspersão, notadamente nos de alta pressão. Desta forma, existe hoje a exigência de se operar com alta eficiência de uso de energia, ou seja, com baixos níveis de desperdício.
Nos estudos da eficiência de irrigação por aspersão, além dos problemas com a uniformidade de distribuição, as perdas de água também reduzem a eficiência do sistema. Segundo Keller \& Bliesner (1990) o excesso de irrigação é, talvez, a maior causa das perdas de água em qualquer sistema de irrigação pois, além do excesso de irrigação devido a um manejo inadequado, as principais causas associadas ao desperdício de água na aspersão

\footnotetext{
${ }^{1}$ Trabalho financiado pela Fundação do Banco do Brasil, com apoio da Usina Sapucaia S.A.

${ }^{2}$ D.Sc. em Eng. Agrícola, Campus Dr. Leonel Miranda, UFRRJ, Campos dos Goytacazes, RJ, Fone: (024) 733 0505, Fax: (024) 723 1324. E-mail: agronomia@ rol.com.br

${ }^{3}$ Professor Titular, CCTA/UENF, Campos dos Goytacazes, RJ, Fone: (024) 726 3752, Fax: (024) 726 3711. E-mail: salassie@uenf.br

${ }^{4}$ Prof. Titular, Departamento Engenharia Agrícola, UFV, Viçosa, MG, Fone: (031) 899 1914/899 1905, Fax: (031) 899 2735. E-mail: mmramos@mail.ufv.br, sediyama@mail.ufv.br

${ }^{5}$ Prof. Adjunto, Departamento de Estatística e Informática, UFV, Viçosa, MG, Fone: (031) 899 1781, Fax: (031) 899 2314. E-mail: cecon@ dpi.ufv.br
} 
são: evaporação das gotas aspergidas e da água da superfície do solo e das folhas, gotas de pequeno diâmetro arrastadas pelo vento (deriva), desperdício nas bordas dos campos irrigados, em vazamentos e no esgotamento da tubulação.

No Brasil, os aspersores de alta pressão têm sido muito utilizados na fertirrigação da cana-de-açúcar com vinhaça, subproduto das destilarias de álcool e na irrigação da cana, em fases específicas de seu crescimento e/ou em períodos de alto déficit hídrico.

Esses sistemas de irrigação expõem o jato pulverizado a grandes alturas (> $7 \mathrm{~m}$ em relação à superfície do solo) o que o torna mais susceptível à deriva das gotas pelo vento e à evaporação da água.

Alguns elementos do clima, como umidade relativa do ar, velocidade do vento etc., podem influenciar a eficiência de irrigação, ocasionando desperdício de energia de bombeamento correspondente à perda de água por evaporação e deriva das gotículas para fora da área irrigada, Frost \& Schwalen (1955), Kraus (1966), Clark \& Finley (1975), Ali \& Barefoot (1981), Dylla \& Shull (1983), Yazar (1984), Paz (1990), Pereira et al. (1995) e Azevedo (1998).

Assim, este trabalho teve como objetivo estudar a influência da velocidade do vento, da umidade relativa do ar, da radiação solar e da pressão de serviço do aspersor no desperdício de energia de bombeamento correspondente às perdas de água produzidas no jato do aspersor.

\section{MATERIAL E MÉTODOS}

Este trabalho foi conduzido na fazenda de propriedade da Usina Sapucaia S.A, no município de Campos dos Goytacazes, RJ; o local é plano e em solo sem cobertura vegetal, latitude de $21^{\circ} 39^{\prime} 22^{\prime}$ 'S, longitude de $41^{\circ} 24^{\prime} 1^{\prime \prime}$ W e altitude de $10,9 \mathrm{~m}$, numa área de cerca de 4,0 ha.

Os testes foram realizados com aspersor-canhão rotativo de impacto, modelo HS 244 B, com diâmetro dos bocais de 35,4 x 10,8 mm e ângulo de inclinação do jato de 24 graus; o aspersor foi instalado sobre um conjunto de saída, composto de saída em aço com suporte de sapatas reguláveis e válvula borboleta de $100 \mathrm{~mm}$ e o bocal de sua saída principal operou, durante os testes, a uma altura de $1,05 \mathrm{~m}$ da superfície do solo, enquanto a água fornecida ao aspersor foi bombeada do canal de irrigação da Usina Sapucaia S.A., com um conjunto moto-bomba a diesel de $120 \mathrm{CV}$ e conduzida através de tubos de $150 \mathrm{~mm}$ de diâmetro.

A vazão do aspersor para cada teste foi determinada por meio de um hidrômetro de turbina horizontal de $150 \mathrm{~mm}$ de diâmetro e, dentro do intervalo das vazões trabalhadas (95,2 a $142,9 \mathrm{~m}^{3} \mathrm{~h}^{-1}$ ) os erros de leitura do hidrômetro, segundo a curva característica do desempenho do hidrômetro fornecido pelo fabricante, eram iguais ou inferiores a $0,3 \%$.

A pressão de serviço do aspersor era determinada no jato do bocal principal, por meio de um manômetro acoplado a um tubo de pitot, realizada imediatamente antes de cada teste. Para monitoramento da pressão durante a realização dos testes usouse o manômetro acoplado à base do aspersor, enquanto os testes foram realizados com um único aspersor trabalhando em pressões que variaram de 318 a $729 \mathrm{kPa}$, e ajustadas por meio de duas válvulas borboleta de $100 \mathrm{~mm}$, sendo uma instalada na saída do conjunto moto-bomba e outra no tubo de subida do aspersor.
A metodologia usada foi aquela descrita pela ASAE (1990). O tempo de operação durante cada teste de aspersor foi de pelo menos 60 min; já a disposição dos pluviômetros para a coleta de água aplicada pelo aspersor foi posicionada numa malha quadrada de $6 \times 6 \mathrm{~m}$.

Utilizaram-se 1156 pluviômetros para coletar a água distribuída pelo aspersor e a medição da água foi realizada com provetas de 500 e $50 \mathrm{~mL}$ de capacidade, usadas conforme o volume coletado.

Os pluviômetros utilizados foram construídos de folha de flandres, com formato cilíndrico, $10 \mathrm{~cm}$ de diâmetro, $15 \mathrm{~cm}$ de altura e apoiados sobre tijolos previamente nivelados junto ao solo; já os dados climáticos usados neste trabalho foram obtidos de uma estação climatológica existente ao lado da área de ensaio.

Antes de se iniciar cada teste, os pluviômetros de um setor radial de abrangência do jato eram virados para baixo, de forma que o jato pudesse permanecer nesse setor para a medição da pressão de serviço do aspersor; após a medida e a leitura inicial do hidrômetro, o teste era começado, colocando-se o aspersor na posição de partida e acionando-se o cronômetro de controle de duração do teste; a seguir, desviravam-se rapidamente os pluviômetros citados, antes que fossem atingidos pelo jato do aspersor; por fim, completava-se o tempo mínimo de $60 \mathrm{~min}$ para finalizar o teste, momento em que se desligava o conjunto motobomba e se fazia a leitura final do hidrômetro.

Para se avaliar a evaporação de água ocorrida durante a leitura dos pluviômetros antes do início de cada teste, instalavamse oito pluviômetros iguais aos utilizados para coleta da água dos aspersores, nos quais eram colocados $50 \mathrm{~mL}$ de água, tampados e posicionados junto da área de teste; após o final do teste do aspersor, os oito pluviômetros controle eram destampados e o volume de quatro deles lido com auxílio de uma proveta de $50 \mathrm{~mL}$; depois, eram feitas as leituras dos pluviômetros que coletaram a água distribuída pelo aspersor e, ao final da leitura dos pluviômetros, eram lidos os quatro pluviômetros controle restantes. Desta forma, obteve-se o volume inicial e final desses pluviômetros para cada ensaio e a evaporação média durante a leitura dos mesmos.

Durante a leitura dos pluviômetros de cada ensaio foram anotados o tempo do início e o final de leitura de cada linha de pluviômetros. Com as informações mencionadas e com as equações de calibração das provetas, realizou-se a correção ponderada da evaporação da água ocorrida durante a leitura de cada linha de pluviômetros.

Estimou-se o desperdício de água $(\mathrm{Da}, \%)$ pela seguinte equação:

$$
\mathrm{Da}=\frac{\mathrm{Va}-\mathrm{Vc}}{\mathrm{Va}} * 100
$$

em que:

Va - volume médio aplicado pelo aspersor, $\mathrm{m}^{3} \mathrm{~h}^{-1}$

$\mathrm{Vc}$ - volume médio coletado, $\mathrm{m}^{3} \mathrm{~h}^{-1}$.

A estimativa do desperdício de energia foi determinada pelo produto das perdas percentuais de água pelo consumo de energia do conjunto eletrobomba expresso em Mega Joule por hectare hora* $\left(\mathrm{MJ} \mathrm{ha}^{-1} \mathrm{~h}^{-1}\right)$ considerando-se o espaçamento entre aspersores, de $72 \times 84 \mathrm{~m}$. O valor de consumo de energia foi calculado por meio da vazão horária, medida em cada teste, e

\footnotetext{
* 1 Mega Joule por ha hora equivale a 0,277725 quilowatt por ha hora ou 0,377498 cavalo vapor por ha hora
} 
das curvas características e de potência da bomba hidráulica, fornecida pelo fabricante (Eqs. 2 e 3, respectivamente) considerando-se o rendimento constante de um motor elétrico genérico de $88 \%$, conforme apresentado por Azevedo Neto (1977). Para a transformação da rotação de $1750 \mathrm{rpm}$ para 1800 rpm, utilizaram-se as equações de Rateaux, recomendadas por Denículi (1993).

A Eq. 2 foi obtida pela análise de regressão, relacionando-se a pressão da bomba (p, em $\mathrm{kPa})$ com a vazão $\left(\mathrm{Q}, \mathrm{em} \mathrm{m}^{3} \mathrm{~h}^{-1}\right)$ para a rotação de $1800 \mathrm{rpm}$

$$
\begin{aligned}
& \mathrm{p}=826,833006+0,837765 \mathrm{Q}-0,007556 \mathrm{Q}^{2} \\
& \left(\mathrm{r}^{2} \text { ajustado }=0,999\right)
\end{aligned}
$$

A Eq. 3 foi obtida pela análise de regressão, relacionando-se os dados de potência do conjunto eletrobomba, considerado ( $\mathrm{P}$ em c.v.) com a pressão (p, em kPa) e vazão da bomba $\left(\mathrm{Q}, \mathrm{em} \mathrm{m}^{3} \mathrm{~h}^{-1}\right)$ para a rotação de $1800 \mathrm{rpm}$.

$$
\begin{aligned}
& \mathrm{P}=-8,055977+0,155577 \mathrm{p}+0,294654 \mathrm{Q} \\
& \quad-0,000107 \mathrm{p}^{2}-5,574715 \mathrm{Q}^{1 / 3} \\
& \mathrm{r}_{\text {ajustado }}^{2}=0,999
\end{aligned}
$$

Eqs. 2 e 3 são válidas para valores de p entre 760 e 840 kPa.

Após a digitação e a preparação dos dados observados no campo, calculou-se o percentual de energia desperdiçada e realizou-se a análise estatística, procedendo-se à análise de regressão múltipla do desperdício de energia, em função das variáveis independentes velocidade do vento, umidade relativa do ar, radiação solar e pressão de serviço do aspersor.

\section{RESULTADOS E DISCUSSÃO}

O desperdício de energia correspondente às perdas de água do jato do aspersor analisado neste trabalho, levou em consideração apenas as perdas de água por evaporação e deriva das gotas do jato do aspersor conjuntamente, sendo que se considerou perdida a água arrastada pelo vento, que não conseguiu atingir o fundo dos pluviômetros. Em outras palavras, toda a água não disponibilizada ao sistema radicular das culturas na área de teste, foi considerada perdida. A deriva das gotículas para fora da área de irrigação por aspersão é considerada, por alguns pesquisadores, Kraus (1966), Thompson et al. (1993) e Thompson et al. (1997) benéfica às culturas localizadas em áreas adjacentes e que tenham suas folhas umedecidas pela água desviada pelo vento; entretanto este benefício é pequeno e sua duração se restringe ao tempo de irrigação somado ao tempo necessário para evaporação do dossel das plantas umedecidas. Segundo Kraus (1966) a lâmina de água depositada em áreas adjacentes à irrigada por sistemas de irrigação por aspersão pode ser considerada desprezível, mas seu efeito sobre o microclima é considerável. Presume-se, pelo exposto, que a água de irrigação arrastada pelo vento não será absorvida pelas raízes da cultura, mas apenas beneficiará temporariamente a cultura pelo microclima criado no dossel das plantas.

Na Tabela 1 são apresentados os resumos das análises das variáveis utilizadas neste trabalho, através de 107 ensaios, para a obtenção do modelo estatístico, enquanto a Eq. 4 foi obtida pela análise de regressão dos resultados de desperdício de energia em $\mathrm{MJ} \mathrm{ha}^{-1} \mathrm{~h}^{-1}$ relacionados ao desperdício percentual de água, em função da velocidade do vento ( $v$ em $\mathrm{m} \mathrm{s}^{-1}$ ), da umidade relativa do ar (UR em \%), da radiação solar (Rs em W m ${ }^{-2}$ ) e da pressão de serviço do aspersor ( $\mathrm{p}$ em kPa). Observa-se, nesta equação, que os efeitos da v, $\mathrm{UR}$, Rs e p sobre o desperdício de energia, foram lineares, sendo seus coeficientes significativos a $1 \%$ de probabilidade, pelo teste " $t$ ", exceção feita ao coeficiente da variável Rs, que foi de $3 \%$ de probabilidade.

Tabela 1. Resumo das análises das variáveis independentes velocidade do vento (v), umidade relativa do ar (UR), radiação solar (RS) e pressão de serviço (p)

\begin{tabular}{lcccc}
\hline \multirow{2}{*}{ Parâmetro } & \multicolumn{4}{c}{ Variáveis } \\
\cline { 2 - 5 } & $\begin{array}{c}\mathrm{V} \\
\left(\mathrm{m} \mathrm{s}^{-1}\right)\end{array}$ & $\begin{array}{c}\mathrm{UR} \\
(\%)\end{array}$ & $\begin{array}{c}\mathrm{RS} \\
\left(\mathrm{W} \mathrm{m}^{-2}\right)\end{array}$ & $\begin{array}{c}\mathrm{p} \\
(\mathrm{kPa})\end{array}$ \\
\hline Média & 3,13 & 66,6 & 511,1 & 558,80 \\
Desvio-padrão & 1,54 & 7,96 & 150,54 & 109,38 \\
Mínimo & 0,53 & 54 & 120 & 317,73 \\
Máximo & 7,17 & 93 & 1025 & 728,63 \\
Observações & 107 & 107 & 107 & 107 \\
\hline
\end{tabular}

$$
\begin{aligned}
& \text { Desperdício }=47,955277+1,506910 \mathrm{v}-0,655991 \mathrm{UR} \\
& +0,007791 \mathrm{Rs}+0,029904 \mathrm{p} \\
& \left(\mathrm{r}^{2} \text { ajustado }=0,76\right)
\end{aligned}
$$

Na Tabela 2 são apresentados os resultados de correlação simples e parcial entre o desperdício de energia e as variáveis independentes utilizadas no modelo estatístico. Observa-se que a maior correlação parcial foi obtida com a variável UR, seguida pela variável p, v e Rs. Observa-se, também, que as variáveis v e Rs apresentaram correlação simples maior que a variável p, porém na correlação parcial mostraram-se inferiores.

Tabela 2. Correlações simples e parcial entre o desperdício de energia e as variáveis independentes velocidade do vento (v), umidade relativa do ar (UR), radiação solar (Rs) e pressão de serviço $(\mathrm{p})$

\begin{tabular}{ccccc}
\hline Correlação & $\mathrm{v}$ & UR & Rs & $\mathrm{p}$ \\
\hline Simples & 0,58 & $-0,71$ & 0,60 & 0,35 \\
Parcial & $-0,44$ & 0,69 & $-0,22$ & $-0,58$ \\
\hline
\end{tabular}

\section{Umidade relativa do ar}

$\mathrm{Na}$ Figura 1 são apresentadas as estimativas do desperdício de energia, em função da UR, para diferentes valores de v, Rs de $520 \mathrm{~W} \mathrm{~m}^{-2}$ e p de $686 \mathrm{kPa}$. Observou-se que o desperdício de energia diminuiu com o aumento da UR. Considerando-se os intervalos de variação das variáveis independentes apresentadas, constatou-se variação no desperdício, de 25,6 $\mathrm{MJ} \mathrm{ha}^{-1} \mathrm{~h}^{-1}$ quando a umidade relativa do ar variou de 54 para 93\%. Esses resultados indicam a grande importância da UR sobre as perdas de água no jato do aspersor, ratificando os resultados de Frost \& Schwalen (1955), Kraus (1966), Seginer \& Kostrinsky (1975), Clark \& Finley (1975), Ali \& Barefoot (1981), Yazar (1984) e Paz (1990). 


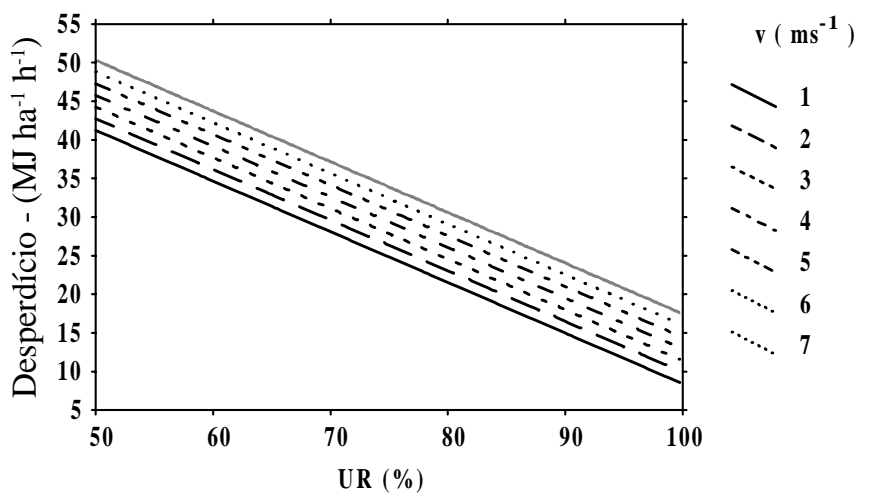

Figura 1. Estimativa do desperdício de energia em função da umidade relativa do ar (UR) para diferentes velocidades do vento (v), radiação solar de $520 \mathrm{Wm}^{-2}$ e pressão de serviço do aspersor de $686 \mathrm{kPa}$

\section{Velocidade do vento}

Na Figura 2 são apresentadas as estimativas do desperdício de energia em função da velocidade do vento $(\mathrm{v})$ para diferentes valores de UR, Rs de $520 \mathrm{Wm}^{-2}$ e p de $686 \mathrm{kPa}$. Constata-se, nesta figura que, quando a velocidade do vento varia de 1 para $7 \mathrm{~m} \mathrm{~s}^{-1}$, para uma UR, o desperdício em energia varia em 9,0 MJ (ha h) ${ }^{-1}$ apresentando relação direta com o desperdício de energia correspondente às perdas de água no jato do aspersor. Esses resultados estão de acordo com os resultados encontrados por Frost \& Schwalen (1955), Kraus (1966), Hermsmeier (1973), Clark \& Finley (1975), Ali \& Barefoot (1981), Dylla \& Shull (1983), Yazar (1984) e Kohl et al. (1987).

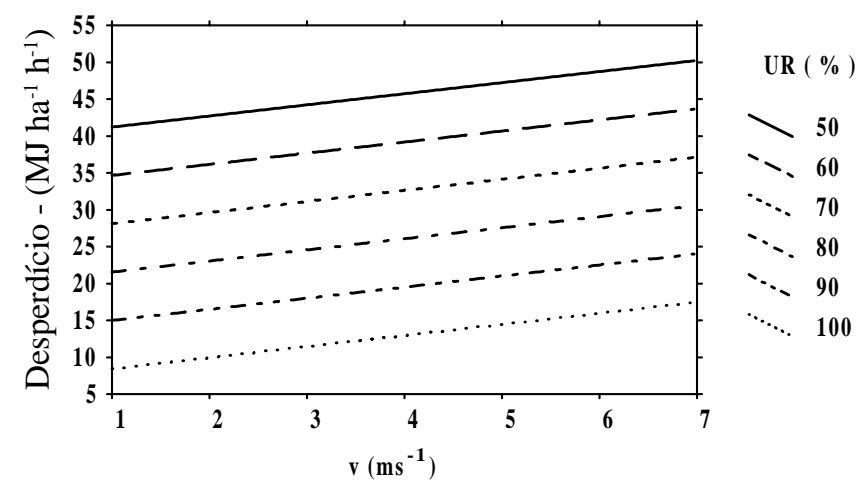

Figura 2. Estimativa do desperdício de energia em função da velocidade do vento (v) para diferentes umidades relativas (UR), radiação solar de $520 \mathrm{Wm}^{-2}$ e pressão de serviço do aspersor de $686 \mathrm{kPa}$

\section{Pressão de serviço do aspersor}

Os resultados de desperdício de energia em função da pressão de serviço do aspersor, apresentados neste trabalho, foram desenvolvidos considerando-se um sistema de irrigação por aspersão do tipo "montagem direta", tal qual se tem usado nas destilarias de álcool para aplicação de vinhaça e/ou água, à cultura da cana-de-açúcar, cuja distância entre o aspersor e o conjunto moto-bomba durante as irrigações varia usualmente, de 80 a 600 m, podendo alcançar até $900 \mathrm{~m}$; assim, a pressão do aspersor varia em função apenas da perda de pressão causada ao longo da tubulação. Desta forma, considerou-se que o conjunto eletrobomba operou sempre a uma rotação de $1800 \mathrm{rpm}$.
Na Figura 3 são apresentadas as estimativas do DMJ ha-1 $\mathrm{h}^{-1}$ em função da pressão de serviço do aspersor (p) para diferentes velocidades do vento, Rs de $520 \mathrm{~W} \mathrm{~m}^{-2}$ e UR de $75 \%$. Constatou-se que o desperdício em energia variou de $12,0 \mathrm{MJ} \mathrm{ha}^{-1} \mathrm{~h}^{-1}$, quando $\mathrm{p}$ variou de 330 a $730 \mathrm{kPa}$. Observa-se que o aumento da pressão ocasionou aumento no desperdício de energia correspondente às perdas de água do jato, devido à maior pulverização do jato aumentando, assim, a sua suscetibilidade à deriva e a evaporação, fato este de acordo com os resultados de Frost \& Schwalen (1955), Seginer (1971), Edling (1985) e Pereira et al. (1995). Com o aumento da pressão, as perdas de água no jato crescem; por outro lado, maiores pressões proporcionam maior uniformidade de distribuição de água, conforme ilustrado na Figura 4, diminuindo assim, as perdas por percolação profunda, aumentando a eficiência de irrigação ocasionada pela melhor distribuição de água. Desta forma, o incremento no desperdício de água no jato, ocasionado pelo aumento de pressão, seria compensado, em parte, pela melhor uniformidade de aplicação de água do aspersor.

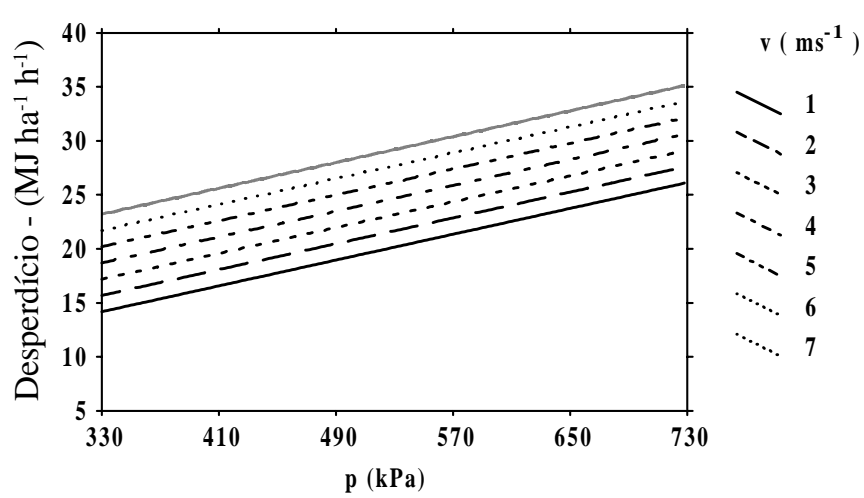

Figura 3. Estimativa do desperdício de energia em função da pressão de serviço do aspersor (p) para diferentes velocidades do vento (v), radiação solar de $520 \mathrm{~W} \mathrm{~m}^{-2}$ e UR de $75 \%$

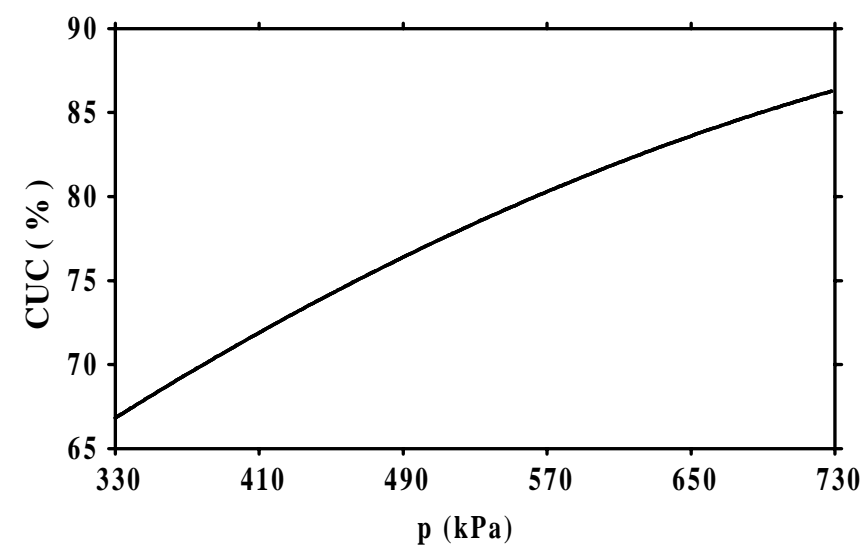

Figura 4. Coeficiente de uniformidade de Christiansen (CUC) em função da pressão de operação do aspersor para $\mathrm{v}$ de $1,0 \mathrm{~m} \mathrm{~s}^{-1}$, direção do vento em relação à linha lateral de $90^{\circ}$, espaçamento entre aspersores de $72 \mathrm{~m}$ e entre linhas laterais, de 84 m (Azevedo, 1998)

\section{Radiação solar}

Poucos trabalhos sobre o desperdício de água e/ou sobre o desperdício de energia decorrente da mesma, foram encontrados utilizando-se a variável radiação solar (Rs); 
entretanto, Seginer \& Kostrinsky (1975) observaram alta correlação total $(r=0.83)$ entre radiação solar e/ou umidade relativa do ar e as perdas de água na irrigação por aspersão, porém os valores de radiação solar utilizados por esses autores, foram estimados e não medidos; além disso, a radiação solar apresenta comportamento similar ao da umidade relativa do ar ao longo do dia, em termos de potencial evaporativo da água, o que causa confundimento entre as duas variáveis. No presente trabalho, apesar da variável Rs ter apresentado resultado estatístico significativo na análise de regressão, a correlação parcial encontrada foi muito baixa $\left(r_{\text {DMJ Rs. UR pv }}=-0.22\right)$. Desta forma, Rs, no presente trabalho, foi considerada variável de menor importância, quando comparada à UR, p e v, tanto em termos de correlação parcial em relação ao desperdício de energia, quanto ao efeito sobre a variação do desperdício.

$\mathrm{Na}$ Figura 5 tem-se as estimativas do desperdício de energia em função da radiação solar para diferentes valores de UR, $v$ de $3 \mathrm{~m}$ $\mathrm{s}^{-1}$ e p de $686 \mathrm{kPa}$. Observa-se que, quando a Rs varia de 120 para $1020 \mathrm{~W} \mathrm{~s}^{-2}$, o desperdício de energia varia em 7,0 $\mathrm{MJ} \mathrm{ha}^{-1} \mathrm{~h}^{-1}$.

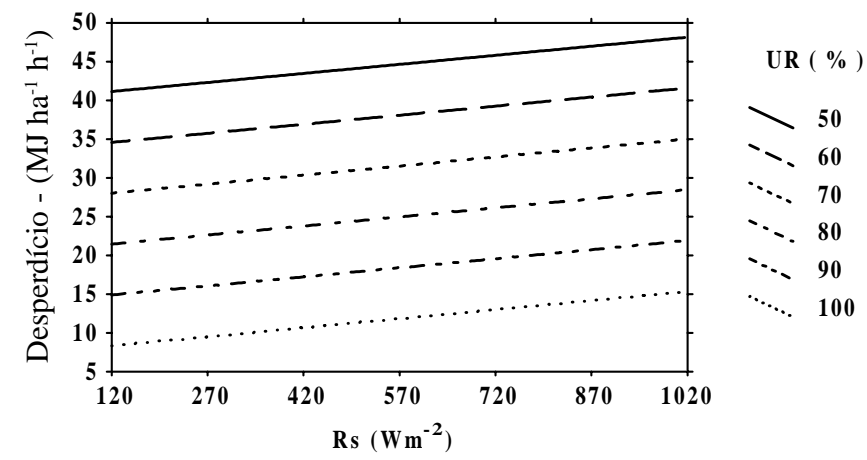

Figura 5. Estimativa do desperdício de energia em função da radiação solar (Rs) para diferentes umidades relativas (UR), velocidade do vento de $3 \mathrm{~m} \mathrm{~s}^{-1}$ e pressão de serviço do aspersor de $686 \mathrm{kPa}$

Na Tabela 3 são apresentados os valores relativos (\%) da influência de cada um dos elementos do clima estudado sobre o desperdício de energia. Os valores percentuais foram calculados tomando-se por base o intervalo de variação entre as condições extremas de desperdício de energia, em que uma das condições foi a de menor demanda evaporativa com UR de $93 \%$, v de $1 \mathrm{~m} \mathrm{~s}^{-1}$, Rs de $120 \mathrm{~W} \mathrm{~m}^{-2}$ para uma pressão de serviço do aspersor constante de $686 \mathrm{kPa}$; a segunda condição foi a de maior desperdício de energia com UR de $54 \%$, v de $7 \mathrm{~m} \mathrm{~s}^{-1}$, Rs de $1020 \mathrm{~W} \mathrm{~m}^{-2}$, para pressão de serviço do aspersor de $686 \mathrm{kPa}$. Calculou-se, a seguir, para cada elemento do clima, o desperdício, utilizando-se o valor do elemento do clima considerado que resultasse no máximo desperdício de energia e, para os demais elementos do clima, considerou-se o valor que ocasionasse o mínimo desperdício; posteriormente, subtraiu-se cada um dos valores do desperdício obtidos pelo valor de desperdício de menor demanda evaporativa, conforme já descrito, resultando nos intervalos de desperdício de energia denominado $\Delta \mathrm{D}$. Com os valores $\Delta \mathrm{D}$ calculou-se a influência relativa de cada um dos elementos do clima considerado em relação ao $\Delta \mathrm{D}$ de maior desperdício de energia. Observa-se, pela tabela, que o desperdício de energia foi mais influenciada pela UR, representando $61,5 \%$ do $\Delta \mathrm{D}$, seguida pela velocidade do vento com $21,7 \%$ e da radiação solar, com $16,8 \%$. A umidade relativa do ar apresentou maior influência sobre o desperdício de energia que os demais elementos do clima, ratificando os resultados de Frost \& Schwalen (1955), Kraus (1966), Seginer \& Kostrinsky (1975), Clark \& Finley (1975) e Yazar (1984). Observa-se, também, que a influência da velocidade do vento não foi tão efetiva no desperdício de energia, correspondente às perdas de água no jato do aspersor, o que está de acordo com Hermsmeier (1973) e Seginer \& Kostrinsky (1975).

Tabela 3. Valores relativos da influência dos elementos do clima no desperdício de energia para uma pressão de serviço do aspersor de $686 \mathrm{kPa}$

\begin{tabular}{lcccccc}
\hline & $\begin{array}{c}\mathrm{UR} \\
\%\end{array}$ & $\begin{array}{c}\mathrm{v} \\
\mathrm{m} \mathrm{s}^{-1}\end{array}$ & $\begin{array}{c}\mathrm{RS} \\
\mathrm{W} \mathrm{m}^{-2}\end{array}$ & $\begin{array}{c}\text { Desperdício } \\
\mathrm{MJ} \mathrm{ha}^{-1} \mathrm{~h}^{-1}\end{array}$ & $\begin{array}{c}\Delta \text { Desperdício } \\
\mathrm{MJ} \mathrm{ha}^{-1} \mathrm{~h}^{-1}\end{array}$ & $\begin{array}{c}\text { Valor } \\
\%\end{array}$ \\
\hline Menor DMJ & 93 & 1 & 120 & 9,90 & 0 & 0 \\
Máximo UR & 54 & 1 & 120 & 35,48 & 25,58 & 61,5 \\
Máximo v & 93 & 7 & 120 & 18,95 & 9,04 & 21,7 \\
Máximo Rs & 93 & 1 & 1020 & 16,92 & 7,01 & 16,8 \\
Maior DMJ & 54 & 7 & 1020 & 51,54 & 41,64 & 100,0 \\
\hline
\end{tabular}

Na Tabela 4 são apresentados os valores relativos (\%) da influência de cada um dos elementos do clima estudados e da pressão de serviço do aspersor no desperdício de energia. Utilizou-se a mesma metodologia apresentada e se observou que $52,3 \%$ do $\Delta \mathrm{D}$ ocorreram em função da $\mathrm{UR}, 18,5 \%$ devido à velocidade do vento, $14,3 \%$ à Rs e $15,0 \%$ devido à pressão.

Tabela 4. Valores relativos da influência dos elementos do clima e da pressão de operação do aspersor no desperdício de energia

\begin{tabular}{lccccccc}
\hline & $\begin{array}{c}\mathrm{UR} \\
\%\end{array}$ & $\begin{array}{c}\mathrm{v} \\
\mathrm{m} \mathrm{s}^{-1}\end{array}$ & $\begin{array}{c}\mathrm{RS} \\
\mathrm{W} \mathrm{m}^{-2}\end{array}$ & $\begin{array}{c}\mathrm{p} \\
\mathrm{kPa}\end{array}$ & $\begin{array}{c}\text { Desperdício } \\
\mathrm{MJ} \mathrm{ha}^{-1} \mathrm{~h}^{-1}\end{array}$ & $\begin{array}{c}\Delta \text { Desperdício } \\
\mathrm{MJ} \mathrm{ha}^{-1} \mathrm{~h}^{-1}\end{array}$ & $\begin{array}{c}\text { Valor } \\
\%\end{array}$ \\
\hline Menor DMJ & 93 & 1 & 120 & 485 & 3,89 & 0 & 0 \\
Máximo UR & 54 & 1 & 120 & 485 & 29,47 & 25,57 & 52,3 \\
Máximo v & 93 & 7 & 120 & 485 & 12,93 & 9,04 & 18,5 \\
Máximo Rs & 93 & 1 & 1020 & 485 & 10,90 & 7,01 & 14,3 \\
Máximo p & 93 & 1 & 120 & 730 & 11,21 & 7,32 & 15,0 \\
Maior DMJ & 54 & 7 & 1020 & 730 & 52,84 & 48,94 & 100,0 \\
\hline
\end{tabular}

\section{CONCLUSÕES}

1. As variáveis que mais influenciaram no desperdício de energia em ordem decrescente, foram a umidade relativa do ar, a velocidade do vento, a pressão de serviço do aspersor e a radiação solar.

2. O desperdício de energia aumentou com a diminuição da umidade relativa do ar e com o aumento da velocidade do vento, da radiação solar e da pressão de operação do aspersor.

\section{REFERÊNCIAS BIBLIOGRÁFICAS}

ALI, S.M.A.; BAREFOOT, A.D. Low trajectory sprinkler patterns and evaporation loss. St Joseph, Michigam: ASAE, 1981. 24 p. Paper, 81-2085

ASAE Prcedure for sprinkler distribution testing for research purposes. Agricultural Engineers Yearbook. St Joseph, Michigan, p. 568-570, 1990. 
AZEVEDO, H.J. de. Influência de elementos do clima no desempenho de sistemas de irrigação por aspersão na região Norte Fluminense. Viçosa MG: UFV. 1998. 145p. Tese Doutorado

AZEVEDO NETTO, J.M. de. Manual de hidráulica. $6^{a}$ ed. São Paulo: Edgrd Blucher LTDA, 1977, cap. 18, p. 250-305.

CLARK, R.N.; FINLEY, W.W. Sprinkler evaporation in the Southern Plains. Chicago, Illinois: ASAE, 1975. 12 p. Paper, 75-2573

DENÍCULI, W. Bombas hidráulicas. Viçosa MG: UFV. Imprensa Universitária, 1993. p. 162.

DYLLA, A.S.; SHULL, H. Estimating losses from a rotatingboom sprinkler. Transaction of the ASAE, St. Joseph, Michigan,v. 26, n. 1, p. 123-125, 1983.

EDLING, R.J. Kinetic energy, evaporation and wind drift of droplets from low pressure irrigation nozzles. Transaction of the ASAE, St. Joseph, Michigan ,v. 28, n. 5, p. 1543-1550, 1985.

FROST, K.R.; SCHWALEN, H.C. Sprinkler evaporation losses. Agriculture Engineering, St Joseph, Michigan v. 36, n. 8, p. 526-528, 1955.

HERMSMEIER, L.F. Evaporation during sprinkler application in a desert climate. In: ANNUAL MEETING AMERICAN SOCIETY OF AGRICULTURE ENGINEERS, Lexington, Kentuchy, 1973. p.1-11. Paper, 73-216

KELLER, J.; BLIESNER, R.D. Sprinkler and trickle irrigation. New York: van Nostrand Reinhold, 1990. 649 p.

KOHL, K.D.; KOHL, R.A.; DeBOER, D.W. Measurement of low pressure sprinkler evaporation loss. Transaction of the ASAE, St. Joseph, Michigan, v.30, n. 4, p. 1071-1074, 1987.
KRAUS, J.H. Application efficiency of sprinkler irrigation and its effects on microclimate. Transaction of the ASAE, St Joseph, Michigan, v. 9, n. 5, p. 642-645, 1966.

PAZ, V.P. Perdas de água e uniformidade de distribuição na irrigação por aspersão. Viçosa: 1990. 59p. Dissertação Mestrado

PEREIRA, G.M.; SOARES, A.A.; ALVES, A.R.; RAMOS, M.M.; MARTINEZ, M.A. Modelo computacional para simulação das perdas de água por evaporação por aspersão. In: CONGRESSO BRASILEIRO DE ENGENHARIA AGRÍCOLA, 24, 1995, Viçosa: Sociedade Brasileira de Engenharia Agrícola

SEGINER, I. Water losses during sprinkler. Transaction of the ASAE, St. Joseph, Michigan, v. 14, n. 5, p. 656-665, 1971.

SEGINER, I.; KOSTRINSKY, M. Wind sprinkler, patterns and system design. Journal of Irrigation and Drainage Division, ASCE, New York, v.101, n. IR4, p. 251-264, 1975.

THOMPSON, A.L.; GILLEY, J.R.; NORMAN, J.M. A sprinkler water droplet evaporation and plant canopy model: II. Model application. Transaction of the ASAE, St. Joseph, Michigan, v. 36, n. 3, p. 743-750, 1993.

THOMPSON, A. L.; MARTIM, D.L.; NORMAN, J.M.; TOLK, J.A.; HOWELL, T.A.; GILLEY, J.R.; SCHNEIDER, A.D. Testing of a water loss distribution model for moving sprinkler systems. Transaction of the ASAE, St. Joseph, Michigan, v. 40, n. 1, p. 81-88, 1997.

YAZAR, A. Evaporation and drift losses from sprinkler irrigation systems under various operating conditions. Agricultural Water Management, Amsterdam, n. 8, p. 439449, 1984. 